\title{
A case report on retroperitoneal PNET/EWS
}

\author{
Zhanqiang Zhang, Honglan Yu, Yingying Shi, Hanping Shi
}

Department of Surgery, The First Affiliated Hospital, Sun Yet-sen University, Guangzhou, China;

${ }^{*}$ Corresponding Author: shihp@,mail.sysu.edu.cn

Received 12 June 2013; revised 20 July 2013; accepted 1 August 2013

Copyright (C) 2013 Zhanqiang Zhang et al. This is an open access article distributed under the Creative Commons Attribution License, which permits unrestricted use, distribution, and reproduction in any medium, provided the original work is properly cited.

\begin{abstract}
Primitive neuroectodermal tumor (PNET) and Ewing's sarcoma (EWS) are small round cell tumors occurring mainly in children and adolescents, rarely in adults. It can occur in multiple tissues and organs including kidney, adrenal, bladder, liver, small intestine, colon and rectum, with a preferred location within the chest area, in the limbs and around the spine, but with rare cases of abdominal, pelvic or retroperitoneal. Here we present a case of the retroperitoneal PENT/EWS in a 38-year-old man and we discuss the clinical features, histopathological characteristics, diagnosis, treatment and prognosis.
\end{abstract}

Keywords: Retroperitoneal; Sarcoma; Primitive Neuroectodermal Tumor; Ewing's Sarcoma

\section{INTRODUCTION}

Primitive neuroectodermal tumor (PNET) and Ewing's sarcoma (EWS) are small round cell tumors occurring in the soft tissues and bones, but rarely in the abdominal cavity, pelvic and retroperitoneal. Here we present a case report on the retroperitoneal PENT/EWS in our hospital, in conjunction with literature review on the topic.

\section{CASE PRESENTATION}

A male subject of 38 years old, who had left upper quadrant mass accompanied with pain for 1.5 years and with enhanced pain since the past 20 days, was admitted into our hospital on July 2, 2007. The subject received left kidney + left retroperitoneal tumor partial resection after revealing a bulky left retroperitoneal tumor by CT and MR examination for his left upper abdominal pain in December 2005 at a local hospital. He then began a chemotherapy program because of the PNET diagnosed by postoperative pathology in January 13, 2006. The chemotherapy regimen was ifosfamide $4.0 \mathrm{~g}$ on day 1-4
+ epirubicin $100 \mathrm{mg}$ on day 1,2 , and 4 . Later, he was admitted into our hospital after a $8 \times 9.6 \mathrm{~cm}^{2}$ cystic space-occupying lesion in his left kidney had been revealed by $\mathrm{CT}$. The results based on consultation on the pathological datasheet collected from the hospital where he received the surgery and chemotherapy indicated that the tumor cells were small and round with strongly stained nuclei, thin chromatin, poorly defined nucleoli, and shrank cytoplasm. The tumor cells were diffused, partly displaying the daisy-like structure. Immunohistochemistry: CD56 and CD99 (+) large-sheet structure tumor cells, CgA and Syn (+) small number tumor cells, LCA (-), EMA and CK (-), vimentin and CD34 (-), Ag tumor cells missing reticular fibers, diagnosed as PENT/ EWS. Laparotomy on May 25, 2006 after appropriate preoperative preparation displayed the left upper quadrant mass up to the diaphragm, down to the left iliac fossa, medial to the right side of the abdominal aorta, lateral to the left paracolic gutter. The lump felt hard and looked smooth and glossy, and was located deep to the waist big muscle closed to spleen. Thus, a complete resection of the whole lump tissue along with the whole spleen and the diaphragm area adhered and infiltrated by the tumor. The patient recovered well after the surgery and was discharged without complications. He continued the same chemotherapy program at the local hospital. He experienced recurrence of left upper quadrant pain 20 days ago. MR displayed at left upper quadrant retroperitoneal tumor; he was then readmitted to our hospital. Physical examination: abdomen slightly bulged, left upper quadrant palpable mass which was ill-defined with tenderness and poor mobility, but without other abnormalities. Upon completion of all necessary preoperative routine examination, no obvious abnormalities were found. The markers for digestive system tumors AFP, CA19-9, CEA, and CA125 were all at normal levels. CT showed a round mass either on the left kidney area or retroperitoneal area, with the upper mass measuring $8 \times 8.9 \times 8.4$ $\mathrm{cm}^{3}$ and the lower one measuring $7 \times 7.1 \times 5.4 \mathrm{~cm}^{3}$; with unclear boundary and adhesion to neighboring tissues, 
close to the diaphragm; the lower lump wrapped iliac artery and adhered to gastrointestinal tract. Otherwise, the patient was in good condition without significant surgical contraindications. After all preoperative preparation, we planned to conduct complete resection of the tumor and its adhesion diaphragmatic with patch repair and graft replacement for the tumor in the lower position had iliac artery adhesions. However, in the morning of the day for operation, the patient had sudden sinus tachycardia with heart rate of $120 \mathrm{bpm}$ at the time of indwelling nasogastric tube. The surgery had to be interrupted. The patients were under monitoring for several days. His heart rate decreased, but remained relatively high compared to baseline heart rate. The patient and his family members asked for provisional discharge. Afterwards, his tumor grew rapidly, but because of the difficult financial situation, he has not received surgery any more. He died of gastrointestinal obstruction, malnutrition, and cachexia 37 days after being discharged.

\section{DISCUSSION}

\subsection{Overview}

PNET and EWS are malignant tumors composed of small round cells, frequently occur in the soft tissue and bone but are very rare clinical cases. They have a common neuroectodermal origin, with similar morphological conformation, specific chromosomeal translocation which is related to the EWS gene on chromosome 22, belonging to the PNET/EWS family tumors [1]. PNET and EWS were once believed to be different types of tumors because of their different differentiations: PNET belongs to neuroectodermal differentiation whereas EWS tends to be undifferentiated. With the advances of immunohistochemistry, electron microscopy, and genetic pathology, bone Ewing's sarcoma, bone Ewing's sarcoma, the peripheral neuroepithelioma or primitive neuroectodermal tumor, Askin's tumor, as well as other types of tumors with their classification still in debate are now all considered as PNET/EWS family tumors [2-4]. A growing number of studies suggest that between PNET and EWS there are no significant differences in terms of their treatment and prognosis. The first case of PNET/EWS family tumors was reported in 1918 by Stout. In recent years, with the development of immunohistochemistry and electron microscopy techniques, our understanding of PNET/EWS family tumors have been gradually deepening and reports on this condition have been steadily increasing. PNET/EWS occurs mainly in children and adolescents, rarely in adults. It can occur in multiple tissues and organs including kidney, adrenal, bladder, liver, small intestine, colon and rectum, with a preferred location within the chest area, in the limbs and around the spine, but with rare cases in abdominal, pelvic or retrop- eritoneal.

\subsection{Clinical Manifestations}

Clinical manifestations depend on the site and the size of the tumor, and the invasion of the surrounding organs. The rapid growth accompanied by pain, lumps, and compression symptoms caused by the tumor is the most common manifestation [5]. Other manifestations include fatigue, weight loss, fever, and various gastrointestinal symptoms such as abdominal distension, nausea, and vomiting.

\subsection{Auxiliary Examination}

There have not been any clinical laboratory tests and specific biomarkers for this type of tumor. B ultrasound, $\mathrm{CT}$, and MR are mainly used to depict tumor internal structure, the scope of violations as well as relations with the surrounding tissue, and meanwhile to provide the information as tumor metastasis, which is of paramount clinical importance for preoperative evaluation of the resection scope and the anticipated efficacy. Imaging often shows soft tissue mass, invasive growth, tendency to invade surrounding tissue, and unclear boundaries with the surrounding tissue structure. In addition to the small size, the tumor generally has uneven density. Under CT, the tumor mostly shows uneven density with visible internal cystic and necrosis, which are worsened by tumor enlargement [6]. If a tumor is seen in the preferred sites with obvious invasion, easy liquefaction and necrosis, as well as incomplete capsule and the separator inside the tumor, especially if when obvious tumor thrombus can be seen in the surrounding intravascular, then the possibility of PNET should be considered [7]. The definite diagnosis still relies on biopsy and pathological examination. To prevent the spread of the tumor and the needle tract transfer, preoperative percutaneous biopsy is generally not recommended, unless the tumor is unresectable and needs radiotherapy and chemotherapy [8].

\subsection{Pathology}

Tumor specimens: oval, lobulated or irregular in shap; infiltrative growth; incomplete capsule, size ranging from $3 \mathrm{~cm} \times 2 \mathrm{~cm} \times 1 \mathrm{~cm}$ to $40 \mathrm{~cm} \times 20 \mathrm{~cm} \times 5 \mathrm{~cm}$; sections are gray or sallow in color, solid and brittle or soft, fish-like hemorrhagic necrosis in texture [9]. Morphological characteristics include malignant tumor mass with small round cells, visible small focal necrosis and spread necrosis under a light microscope, which may or may not form the Homer-Wright rosettes. CD99 (MIC2) is uniformly and diffusely expressed on the cell membrane of the tumor, and vimentin, NSE, Syn, of Cag, NF, and S-100 are also expressed to varying abundances. 
Glycogen granules or nerve secretory granules can be seen under an electron microscope $[9,10]$.

From the molecular pathological view, PNET/EWS have the same $\mathrm{t}(11 ; 22)$ (q24; q12), or less $\mathrm{t}(21 ; 22)$ (q22; $\mathrm{q} 12)$ or $\mathrm{t}(7 ; 22)(\mathrm{p} 22 ; \mathrm{q} 12)$. All these chromosomal rearrangements fuse the EWS gene on chromosome 22 to FLI21 ERG or ETV1 gene members of the ETS transcription factor family, resulting in EWS/FLI21, EWS/ ERG or EWS/ETV1 fusion genes, leading to tumorigenesis. $95 \%$ of the tumors can be detected by RT-PCR and FISH with high sensitivity and specificity [11].

Identification and diagnosis of this type of tumor primarily relies on comparisons with other intra-abdominal and retroperitoneal soft tissue tumors, including the location, age, tumor morphological alterations, immunohistochemical illustration, and genetic traits.

\subsection{Treatment and Prognosis}

PNET/EWS is a systemic disease and its therapy requires comprehensive treatment, including surgery, radiotherapy, chemotherapy and biological therapy. There is no uniform regimen for intraperitoneal or retroperitoneal PNET/EWS. Rud et al. [12] and Covelli et al. [13] suggested that the surgery may be more important for EWS outside bone than EWS in the bone and total resection yields better prognosis. Based on the literature, complete surgical removal may be the most effective means for cure; even for patients with local recurrence, if possible, surgical treatment should be implemented. In order to ensure complete resection, removal of some adjacent organs, the most commonly kidney, adrenal, colon, pancreas, and spleen, is often necessary. With the development of vascular surgery, resection of involved vessels and implantation of artificial blood vessels may be coapplied, though the long-term efficacy and benefits await further studies to confirm.

As far as radiotherapy and chemotherapy, there are different views. Kinsella et al. [14] believed that high doses of local radiotherapy and VAC procedures are adequate for EWS outside bone and total surgical resection may be unnecessary. Local adjuvant radiotherapy after complete resection cannot significantly reduce the local recurrence because of high doses and side effects that require stringent protective measures $[12,15]$. Venkitaraman et al. [16] proposed that intensive chemotherapy is necessary after complete resection of the tumor or radiotherapy with doses $>50 \mathrm{~Gy}$, for patients with PNET/ EWS sarcoma outside bone in order to obtain good effect. Of 14 PNET/EWS patients without metastases who underwent combinational chemotherapy (5 with VAC procedures, 5 with VACA procedures, four with VAC/IE procedures), 8 were cured, 2 partially cured, and 4 had progressive deterioration [16].

Venkitaraman et al. [16] believed that high hemoglo- bin, low lactate dehydrogenase, VAC/IE chemotherapy, and complete response to chemotherapy are the factors for raising tumor-free survival rate and overall survival rate. Martin et al. [15] considered the initial performance of tumor (primary or recurrent) is an important prognostic factor, whereas tumor location, size, and age make no statistical significance $[15,16]$. PNET/EWS patients without metastatic who received combination therapy had 5-year survival rate of $60 \%$ and overall survival rate of $30 \%$ [16]. Aggressive PNET/EWS causes distant metastases at early stages, frequent local recurrence and regional lymph nodes, lungs, liver, bone and bone marrow metastasis, and has poor prognosis with the median survival time of about two years; the leading cause of death is metastasis to the lung, bone, liver and other organs.

\section{CONCLUSION}

The abdominal cavity and retroperitoneal PNET/EWS are relatively rare, but they grow rapidly in larger size, oppress surrounding organs and large vessels, and make surgical resection difficult. It is often in high degree of malignancy and already has widespread subclinical metastasis at the time of going to a clinic. Moreover, it can quickly transfer to everywhere throughout the body rendering poor prognosis. Clinicians should be highly alert to this condition. Imaging is the primary means of early detection and early diagnosis. Complete surgical resection may be the most effective way of cure. Whenever possible, surgery should be implemented even in patients with local recurrence. In order to ensure complete resection, removal of any neighboring organs, most commonly kidney, adrenal, colon, pancreas, spleen, and so on, may be required. With the development of vascular surgery, resection of involved vessels and implantation of artificial blood vessels may be conducted. The efficacy of radiotherapy is still unclear. Future studies are warranted to develop surely effective surgical treatment options and preoperative/postoperative adjuvant therapies.

\section{REFERENCES}

[1] Parham, D.M., Roloson, G.J., Feely, M., Green, D.M., Bridge, J.A. and Beckwith, J.B. (2001) Primary malignnant neuroepithelial tumors of the kidney: A clinicopathologic analysis of 146 adult and pediatric cases from the National Wilms' Tumor Study Group Pathology Center. American Journal of Surgical Pathology, 25, 133-146. doi:10.1097/00000478-200102000-00001

[2] Lopez-Terrada, D. (1996) Molecular genetics of small round cell tumors. Seminars in Diagnostic Pathology, 13, 242-249.

[3] Delattre, O., Zucman, J., Melot, T., Garau, X.S., Zucker, J.M., Lenoir, G.M., Ambros, P.F., Sheer, D., Turc-Carel, C. and Triche, T.J. (1994) The Ewing family of tumors-A 
subgroup of small-round-cell tumors defined by specific chimeric transcripts. New England Journal of Medicine, 331, 294-299. doi:10.1056/NEJM199408043310503

[4] Grier, H.E. (1997) The Ewing family of tumors: Ewing's sarcoma and primitive neuroectodermal tumors. Pediatric Clinics of North America, 44, 991-1004. doi:10.1016/S0031-3955(05)70541-1

[5] Nikitakis, N.G., Salama, A.R., O’Malley Jr., B.W., Ord, R.A. and Papadimitriou, J.C. (2003) Malignant peripheral primitive neuroectodermal tumor-peripheral neuroepithelioma of the head and neck: A clinicopathologic study of five cases and review of the literature. Head \& Neck, 25, 488-498. doi:10.1002/hed.10260

[6] Zhang, W.D., Xie, C.M., Mo, Y.X. and Li, J.Y. (2007) CT and MRI features of peripheral primitive neuroectodermal tumor. Chinese Journal of Cancer, 26, 643-646.

[7] Xiao, Q., Gu, Y.J. and Yang, T.C. (2004) CT and MRI evaluation of peripheral primitive neuroectodermal tumors. China Oncology, 14, 333-338.

[8] Mendenhall, W.M., Zlotecki, R.A., Hochwald, S.N., Hemming, A.W., Grobmyer, S.R. and Cance WG. (2005) Retroperitoneal soft tissue sarcoma. Cancer, 104, 669-675. doi:10.1002/cncr.21264

[9] Zhang, Z.H., Fan, Q.H., Xu, T.R. and Zheng, Z.X. (2001) Clinicopathology of peripheral primitive neuroectodermal tumor/Ewing's sarcoma. Chinese Journal of Clinical and Experimental Pathology, 17, 306-309.

[10] He, L.J., Li, P.J., Liu, S.R., Wang, L., Liu, N., Du, X.Y. and Lang, Z.Q. (2000) Peripheral primitive neuroectoder- mal tumor and Ewing's sarcoma: Clinicopathological and immuneohistochemical studies. Chinese Journal of Diagnostic Pathology, 7, 249-251.

[11] Zhang, Z.H. (2002) New progress in molecular pathology of peripheral primitive neuroectodermal tumor and Ewing's sarcoma. Foreign Medical Sciences (Pathophysiology and Clinical Medicine), 22, 191-193.

[12] Rud, N.P., Reiman, H.M., Pritchard, D.J., Frassica, F.J. and Smithson, W.A. (1989) Extraosseous Ewing's sarcoma. A study of 42 cases. Cancer, 64, 1548-1553. doi:10.1002/1097-0142(19891001)64:7<1548::AID-CNC R2820640733>3.0.CO;2-W

[13] Covelli, H.D., Beekman, J.F. and Kingry, R.L. (1980) Extraskeletal Ewing's sarcoma: Prolonged survival with recurrence after operation. Southern Medical Journal, 73, 1294-1295. doi:10.1097/00007611-198009000-00053

[14] Kinsella, T.J., Triche, T.J., Dickman, P.S., Costa, J., Tepper, J.E. and Glaubiger, D. (1983) Extraskeletal Ewing's sarcoma: Results of combined modality treatment. Journal of Clinical Oncology, 1, 489-495.

[15] Martin II, R.C. and Brennan, M.F. (2003) Adult soft tissue Ewing sarcoma or primitive neuroectodermal tumors: Predictors of survival? Archives of Surgery, 138, 281-285. doi:10.1001/archsurg.138.3.281

[16] Venkitaraman, R., George, M.K., Ramanan, S.G. and Sagar, T.G. (2007) A single institution experience of combined modality management of extra skeletal Ewings sarcoma. World Journal of Surgical Oncology, 5, 1-4. doi:10.1186/1477-7819-5-3 